\title{
Flavour independent Higgs searches at LEP-2
}

\author{
P. Bambade \\ Laboratoire de l'Accélérateur Linéaire \\ IN2P3/CNRS et Université Paris-Sud - Bât. 200 - BP 34 - 91898 Orsay cedex, France
}

\begin{abstract}
Searches for hadronically decaying neutral Higgs bosons, conducted at LEP-2 in the framework of the Standard and Minimal SuperSymmetric Models, strongly exploit the information from its $b \bar{b}$ decays. However, although this signature dominates the accessible mass range in these models, there are special cases of extended models with suppressed $h \rightarrow b \bar{b}$ couplings. Recently, complementary searches, performed without relying on $b$-quark identification, have enabled to reduce the model-dependence of the results, for both $h Z$ and $h A$ production mechanisms. The preliminary results obtained by the four LEP collaborations are presented. No evidence for a signal was found. Flavour independent bounds on Higgs boson production are given, and used to constrain specific scenarios.
\end{abstract}

Talk given at the $10^{\text {th }}$ Lomonosov Conference on Elementary Particle Physics, Moscow (Russia), August 23-29, 2001. 


\section{Introduction}

There are extensions of the Standard Model (SM) in which Higgs bosons have suppressed couplings into $b$-quarks. This can occur for specific parameters of the Two Higgs Doublet Model (2HDM) [1], or of the Minimal SuperSymmetric Model (MSSM) [2], as well as for some composite models [3]. Searches for SM and MSSM neutral Higgs bosons, produced through $e^{+} e^{-} \rightarrow h Z, h A$ processes, would have reduced sensitivities in such cases, because of their strong reliance on the identification of $b$-quarks from the Higgs boson decays to maximize the separating power $[4,5,6]$. It is very important to also cover such scenarios experimentally, with dedicated searches not exploiting the b-tagging, to reduce the model-dependence of the results.

All LEP collaborations have developed versions of their Higgs searches without $b$-tagging in recent years. The data collected in 1998, 1999 and 2000, at centre-of-mass energies near $189 \mathrm{GeV}$, and in the ranges 192-202 and 204-208 GeV, respectively, were analysed. No evidence for a signal was found. Results were given in terms of cross-section or mass exclusions, independent of the flavour of the Higgs boson decays $[7,8,9,10,13,14]$, and were also combined with results from other Higgs boson searches to constrain specific models $[6,11,12,15]$.

In this report, the main features of these new analyses are described, highlighting aspects specific to the flavour independent hypothesis. The obtained results are presented, and model independent interpretations, as well as applications to specific scenarios, are discussed.

\section{Search for $h Z$ production}

\subsection{Analysis}

The data collected by the four LEP experiments at the highest energies were used to search for $h Z$ production in the flavour independent hypothesis. The analysis methods were adapted from existing LEP-2 searches or measurements performed in the four-jet $(q \bar{q} q \bar{q})$, missing energy $(q \bar{q} \nu \bar{\nu})$ and leptonic $\left(q \bar{q} l^{+} l^{-}\right)$topologies ${ }^{1}$. In all channels except four-jets, events were selected exactly, or almost exactly, as in the corresponding SM Higgs boson search, removing the $b$-tagging from the final selection. In the four-jet channel, on the other hand, all collaborations used dedicated test-mass dependent selections, to exploit maximally the specific kinematic features and the mass reconstruction. This was necessary to reduce dominant backgrounds from $W W$ and $Z Z$ production and from QCD multi-jet processes, without using $b$-tagging information.

ALEPH [7] used the data collected in 1998-2000, clustered in seven energy bins in the last year. In the four-jet channel, a dedicated search based on a neural network method was developed. The compatibility of the data with the signal hypothesis was tested in the Higgs boson mass range from 60 up to $115 \mathrm{GeV} / \mathrm{c}^{2}$, in $5 \mathrm{GeV} / \mathrm{c}^{2}$ steps, interpolating the final discriminant variable for intermediate values. DELPHI [8] used the data collected in 1999-2000, clustered in two energy bins in the last year. The compatibility of the data with the signal hypothesis was tested in the Higgs boson mass range from 50 up to $110 \mathrm{GeV} / \mathrm{c}^{2}$, in $5 \mathrm{GeV} / \mathrm{c}^{2}$ steps. A small reduction in performance was introduced in the final evaluation, between each test mass, to account for mass resolution effects. L3 [9] used the data collected in 1999-2000, clustered in five energy bins in the last year. OPAL [10] used the data collected in 1998-2000, clustered in a single energy bin in the last year. In L3, and for the four-jet channel in OPAL, test-masses were chosen in the Higgs boson mass range from $60 \mathrm{up}$ to $115 \mathrm{GeV} / \mathrm{c}^{2}$, in $1 \mathrm{GeV} / \mathrm{c}^{2}$ steps. All topologies corresponding to possible decay products of the $Z$ boson were analysed by all collaborations, except, in the case of DELPHI, $q \bar{q} \tau^{+} \tau^{-}$.

Even though $b$-tagging was not used, every experiment did find some variation in performance between the possible decay products of the Higgs boson, arising from differences in mass resolution and jet structure ${ }^{2}$.

\footnotetext{
${ }^{1}$ Throughout the paper, the notation $q$ stands for both primary quarks or gluons produced in Higgs boson decays.

${ }^{2}$ For instance, Higgs boson decays into gluon pairs have larger multiplicities, but at the same time coarser dijet mass resolution, than decays into light quarks.
} 
In order to enable quoting genuinely flavour independent results, the samples used for the final evaluation were therefore conservatively chosen as those which gave the weakest expected performance in each channel, and for each value of the Higgs boson mass.

In general, because these searches were developed recently, they were up to now less optimised, slightly less comprehensive, and used more simplifying assumptions to extract the results than corresponding searches for SM or MSSM Higgs bosons. Also, although potential biases to the results were studied by individual experiments, there was no consideration yet of systematic effects in the common evaluation. All results presented here are preliminary.

\subsection{Results}

No evidence for a signal was found in any of the topologies analysed by the four collaborations. The information from all topologies and collaborations was combined to compute flavour independent bounds on $h Z$ production as a function of the Higgs boson mass [14]. The standard statistical procedures based on the Modified Frequentist approach and using the likelihood ratio technique [16] were applied as in the other combinations performed by the LEP working group for Higgs boson searches [5]. The observed and expected confidence levels for the signal, $C L_{s}$ and background-only, $C L_{b}$ hypotheses, obtained for each of the four collaborations, using two independent implementations of the combination software [18, 17], were very consistent with results obtained within each collaboration. The calculations of the observed and expected 95\% CL lower limits on the mass of the Higgs boson, assuming production cross-sections equal to those in the SM and $100 \%$ hadronically decaying Higgs bosons, are shown from one of the implementations[18] in Table 1, together with the combined results using the data from all four collaborations ${ }^{3}$. The combined observed and median expected limits were 112.9 and $113.0 \mathrm{GeV} / \mathrm{c}^{2}$, respectively.

\begin{tabular}{|c|c|c|}
\hline Collaboration & Obs. limit $\left(\mathrm{GeV} / \mathrm{c}^{2}\right)$ & Exp. median limit $\left(\mathrm{GeV} / \mathrm{c}^{2}\right)$ \\
\hline ALEPH & 109.3 & 108.4 \\
DELPHI & 109.6 & 108.8 \\
L3 & 111.6 & 109.3 \\
OPAL & 109.4 & 108.5 \\
\hline \hline LEP & $\mathbf{1 1 2 . 9}$ & $\mathbf{1 1 3 . 0}$ \\
\hline
\end{tabular}

Table 1: Flavour independent observed and expected 95\% CL lower limits on the mass of the Higgs boson, assuming production cross-sections equal to those in the SM and $100 \%$ hadronically decaying Higgs bosons. Systematic uncertainties were not included.

The confidence levels $C L_{s}$ and $C L_{b}$ obtained from the full combination in the signal and background-only hypotheses are shown as a function of the mass in Figure 1. Good overall agreement can be seen between the observation and expectation in the absence of a signal. The slightly lower values for $1-C L_{b}$ for masses below $80 \mathrm{GeV} / \mathrm{c}^{2}$ reflect an excess of data in this region, and may be the result of some systematic effect. An investigation is in progress. A 5 sigma discovery corresponds to a value of $1-C L_{b}$ of $5.710^{-7}$, as indicated by the horizontal line. The sensitivity for such a discovery is reached for an assumed Higgs boson mass of $107 \mathrm{GeV} / \mathrm{c}^{2}$, when this line is intersected by the expected median confidence in the background-only hypothesis.

\footnotetext{
${ }^{3}$ Because the evaluations performed did not include systematic uncertainties, the values obtained differ in some cases from those quoted by the individual collaborations which included them in their evaluations. This is most notably the case for the OPAL results [10].
} 

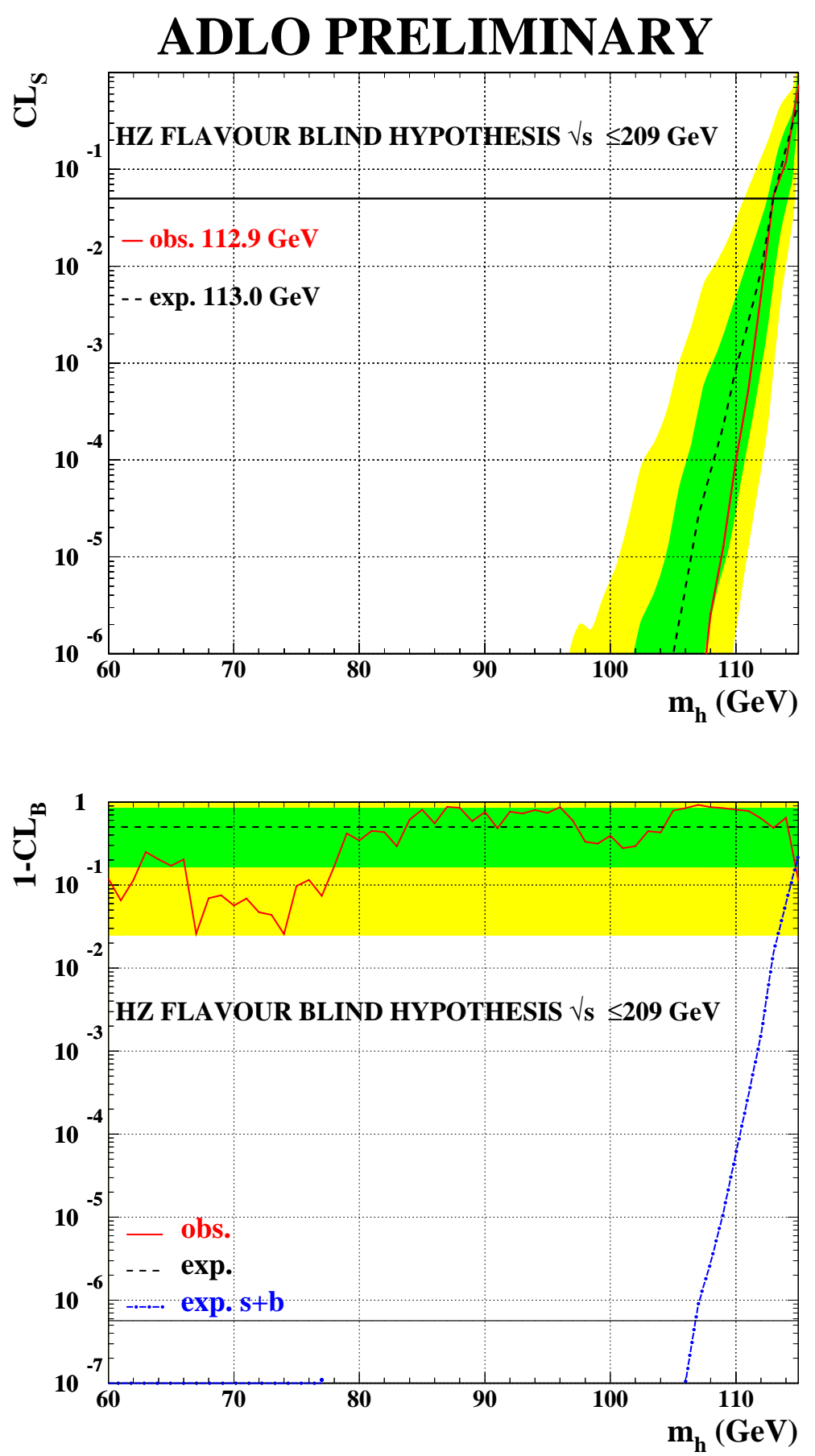

Figure 1: Combined LEP confidence levels as a function of the Higgs boson mass in the flavour independent hypothesis, assuming production cross-sections equal to those in the SM and $100 \%$ hadronically decaying Higgs bosons. The confidence levels for the signal and background-only hypotheses are shown in the upper and lower plots, respectively. The curves are the observed (solid) and expected median (dashed) confidences from background-only experiments, and the bands are the corresponding 68.3 and $95 \%$ confidence intervals. In the lower plot, the dot-dashed line shows the expected median confidence from experiments including an expected signal of mass given in abscissa. 


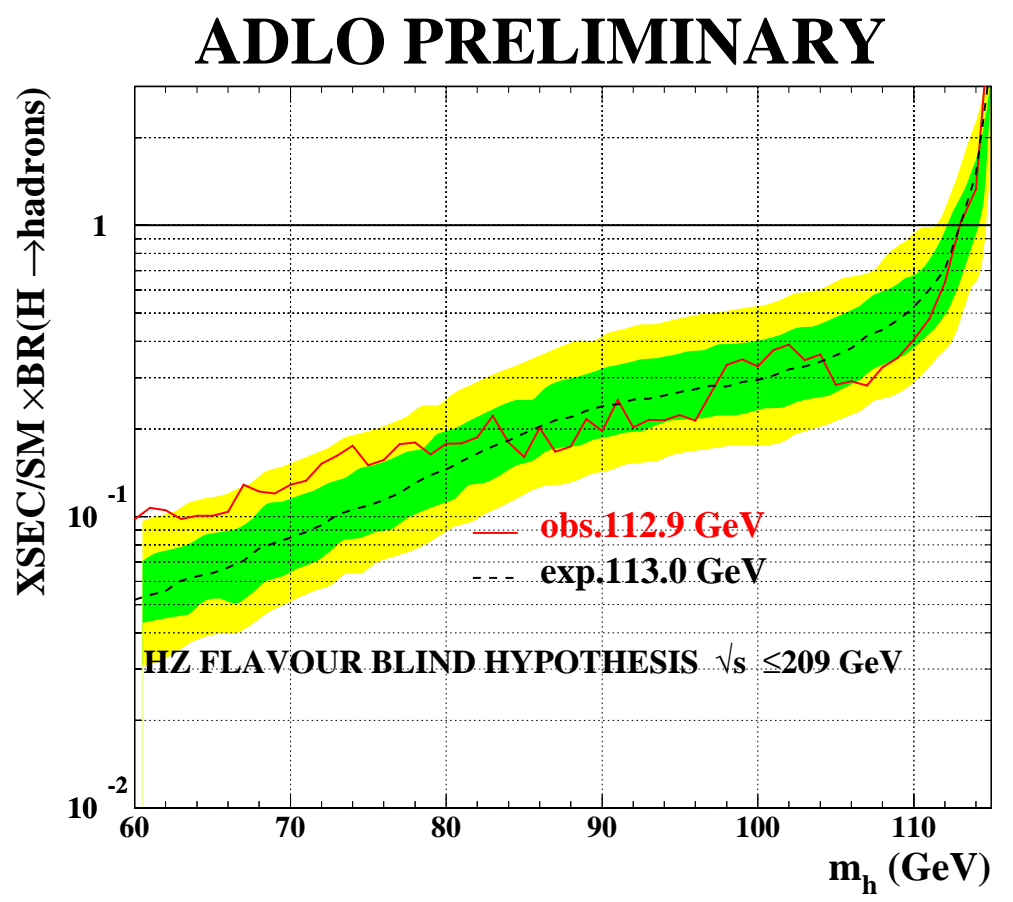

Figure 2: Combined flavour independent LEP observed and expected 95\% CL upper limits on the production cross-section as a function of the Higgs boson mass, normalised to the expected SM values, computed assuming 100\% hadronically decaying Higgs bosons. The curves are the observed (solid) and expected median (dashed) excluded ratios, and the bands correspond to 68.3 and $95 \%$ confidence intervals from the background-only experiments.

Upper limits on the production cross-section as a function of mass were also determined for each individual collaboration, and combined using the data from all four collaborations. Typically, crosssections larger than about $10-60 \%$ of the expected SM value were excluded at $95 \%$ CL in the mass range $60-100 \mathrm{GeV} / \mathrm{c}^{2}$, by each collaboration alone. The combined exclusion from the four collaborations is shown as a function of the mass in Figure 2. In the same mass range, it is possible to exclude cross-sections larger than about a few to $30 \%$ of the expected SM value with the full LEP-2 data set. Good overall agreement between the observation and expectation can be seen also here, except in the lower mass range, where the weaker observed limit is resulting from some excesses of data in this region, as was already noted.

\section{$3 \quad$ Search for $h A$ production}

The search for $h A$ production used hadronic final states [8,11, 12], associated or not with $\tau^{+} \tau^{-}$leptons [13]. No signal was found. Flavour independent results were obtained separately by each collaboration, in terms of excluded ranges for $h$ and $A$ masses, for different assumptions on production cross-sections $[8,13]$. Only part of the available data was used so far, and systematic effects were not considered in all the studies. Moreover, some selections used were not fully optimised. No LEP-wide combination of results was performed yet. All results except [12] are preliminary. Applications to constrain specific models, through combination $[6,11,12$, 15] with other Higgs boson searches, both standard and flavour independent, are discussed in the following two sections. 


\section{$3.1 \quad$ DELPHI $[8]$}

The DELPHI flavour independent $h A$ search used most of the data collected in 1998-2000. Only fully hadronic $(q \bar{q} q \bar{q})$ final states were analysed. In order to cover a wide range of masses (in the range $m_{h}, m_{A}>$ $15 \mathrm{GeV} / \mathrm{c}^{2}$ and $40<m_{h}+m_{A}<180 \mathrm{GeV} / \mathrm{c}^{2}$ ) three complementary analysis streams were defined, for fourjet, three-jet and three-jet-with-high-thrust topologies. A test-mass dependent discriminant variable based on the mass reconstruction was obtained in each stream. Signal samples were generated in $10 \mathrm{GeV} / \mathrm{c}^{2}$ steps for $m_{h}$ and $m_{A}$, if both masses were above $30 \mathrm{GeV} / \mathrm{c}^{2}$, and $5 \mathrm{GeV} / \mathrm{c}^{2}$ if one was below. In the final evaluation, the discriminant variable was interpolated to test intermediate values, using $2 \mathrm{GeV} / \mathrm{c}^{2}$ steps, and the overlap between analysis streams was removed by choosing the strongest one, based on expected performance. The efficiencies obtained were checked to be flavour independent at the $3 \%$ level. The procedure described in [19] was applied to compute $95 \% \mathrm{CL}$ exclusions in the $\left(m_{h}, m_{A}\right)$ plane (see Figure 3), assuming maximal $Z h A$ couplings ${ }^{4}$ and $100 \%$ hadronically decaying $h$ and $A$ bosons.

\section{$3.2 \quad$ L3 [13]}

The L3 flavour independent $h A$ search used the data collected in 1998-1999, focusing on the range $m_{h}, m_{A}>$ $50 \mathrm{GeV} / \mathrm{c}^{2}$. Both $q \bar{q} q \bar{q}$ and $q \bar{q} \tau \bar{\tau}$ final states were analysed. Test-mass dependent selections were constructed, based on kinematics and mass reconstruction, to compute $95 \%$ CL exclusions in the $\left(m_{h}, m_{A}\right)$ plane, for several assumptions on the $h A$ production cross-section.

\subsection{OPAL $[11,12]$}

The OPAL flavour independent $h A$ search used the data collected in 1998. Only fully hadronic $(q \bar{q} q \bar{q})$ final states were analysed. A test-mass dependent discriminant variable based on kinematics and mass reconstruction was used to evaluate the compatibility of data and simulation for a fixed set of mass hypotheses in the range $m_{h}, m_{A}>30 \mathrm{GeV} / \mathrm{c}^{2}$. Final selection efficiencies were interpolated to test intermediate values, using the most conservative final-state flavour assignment. The results were not expressed directly in terms of bounds on $h A$ production, but combined with other Higgs boson searches to provide general limits in the context of the $2 \mathrm{HDM}$ (see the next section).

\section{Constraints on Two Higgs Doublet Models of Type II}

The popular $2 \mathrm{HDM}[1]$ provides an attractive framework for improving the SM because it features the simplest possible extension of the Higgs sector satisfying the constraints of $\rho \simeq 1$ and the absence of treelevel flavour changing neutral currents ${ }^{5}$, and has few new parameters. In $2 \mathrm{HDMs}$, tree-level production cross-sections for neutral Higgs bosons $h$ and $A$ are related to SM expressions via

$$
\sigma_{h Z}=\sin ^{2}(\beta-\alpha) \sigma_{H Z}^{S M} \text { and } \sigma_{h A}=\cos ^{2}(\beta-\alpha) \lambda \sigma_{H Z}^{S M},
$$

where the phase-space factor $\lambda$ accounts for the $p$-wave threshold behaviour, $\alpha$ is the Higgs mixing angle, and $\tan \beta$ the ratio of vacuum expectation values for the two Higgs superfields. Couplings to fermions specify the type of $2 \mathrm{HDM}$. In Type II $2 \mathrm{HDMs}$, the first Higgs doublet couples only to down-type fermions, and the second only up-type fermions. In this case, tree-level couplings between neutral Higgs bosons $h, A$ and

\footnotetext{
${ }^{4}$ The $e^{+} e^{-} \rightarrow h A$ production cross-sections were computed with the HZHA generator [20], taking into account the kinematical suppression in the phase-space from the $p$-wave threshold behaviour, and effects from initial state radiation, but specifying $\cos ^{2}(\beta-\alpha)=1$ in the expression of the $Z h A$ coupling (see section 4 for a description of couplings in the 2HDM).

${ }^{5}$ If Higgs-fermion couplings are appropriately chosen.
} 


\section{DELPHI PRELIMINARY}

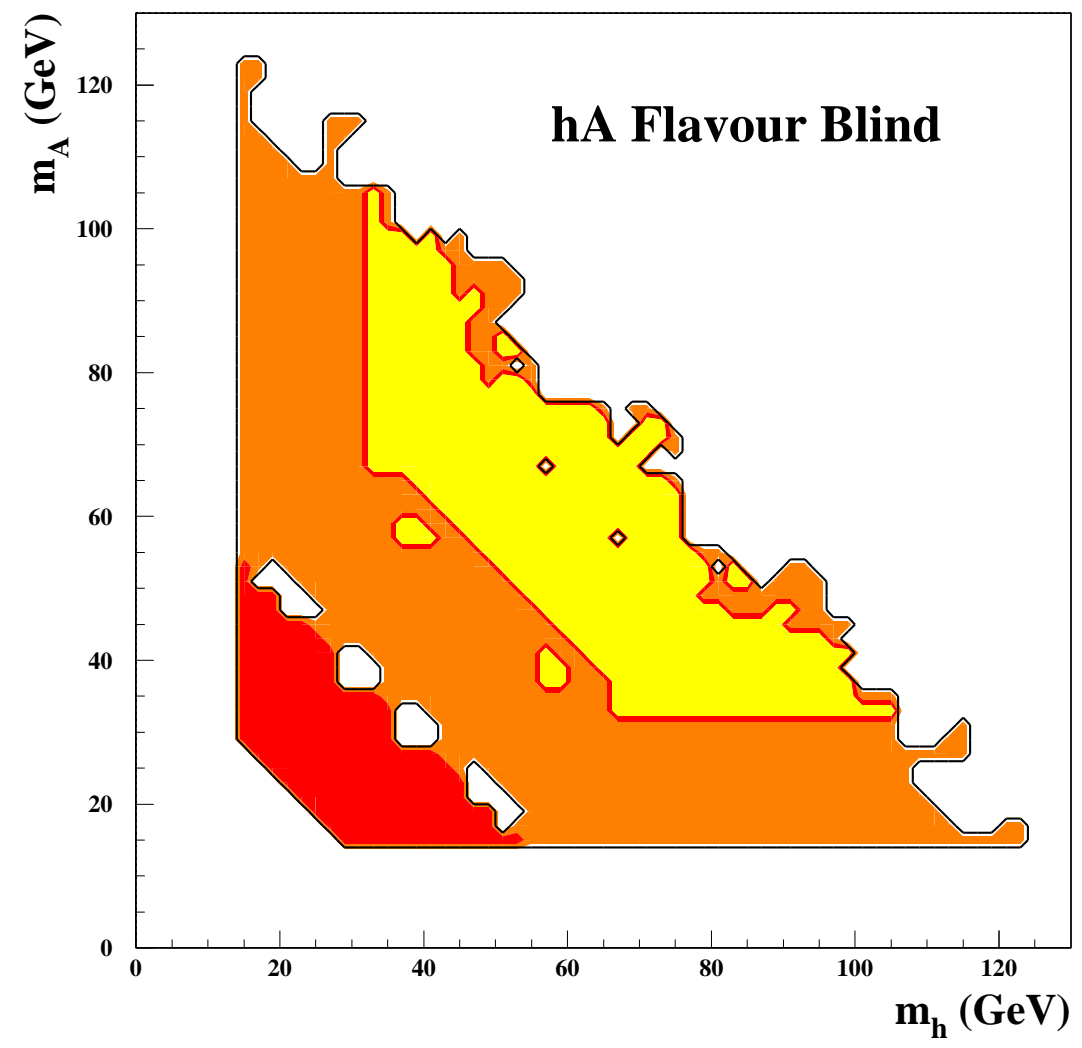

Figure 3: Flavour independent 95\% CL excluded ranges in the $\left(m_{h}, m_{A}\right)$ plane, assuming maximal $Z h A$ couplings and $100 \%$ hadronically decaying $h$ and $A$ bosons, shown using lighter to darker grey for the four-jet, three-jet and three-jet-with-high-thrust streams, respectively. The unexcluded region near the $m_{h}+m_{A}=70$ $\mathrm{GeV} / \mathrm{c}^{2}$ line is due to the transition between three-jet and three-jet-with-high-thrust streams, which is still being optimised.

fermions are related to SM couplings through the factors

$$
h c \bar{c}: \frac{\cos \alpha}{\sin \beta}, \quad h b \bar{b}:-\frac{\sin \alpha}{\cos \beta}, \quad A c \bar{c}: \cot \beta \quad \text { and } \quad A b \bar{b}: \tan \beta .
$$

The MSSM is a physically strongly motivated Type II 2HDM. New particles and specific constraints on model parameters arise in this case from the introduction of supersymmetry, and also once specific schemes for its breaking are added.

However, if full generality is sought, no such constraints can be invoked, and parameters such as $\alpha, \beta, m_{h}, m_{A}$ are entirely free. In large regions of the $(\alpha, \beta)$ parameter space, suppressed production of either $h$ or $A$ can occur, together with suppressed decay rates into $b \bar{b}$, for values of $m_{h}, m_{A}$ kinematically accessible at LEP-2. General model-independent bounds can in this context only be obtained from broad scans of the $\left(\alpha, \beta, m_{h}, m_{A}\right)$ parameter space, by combining results from standard and flavour independent $h Z$ and $h A$ searches. 
This has been done by OPAL $[11,12]$. The best illustration of the results is shown in Figure 4 , where $95 \% \mathrm{CL}$ excluded ranges in the $\left(m_{h}, m_{A}\right)$ plane are given for almost any $\alpha, \beta$. Although weaker than in more constrained scenarios [6], the excluded mass ranges obtained are significant given their high level of generality. Even if full exclusion remains out of reach in this approach, specific hypotheses (theoretically motivated or driven by independent observations), corresponding to scenarios or ranges of parameters of the Type II $2 \mathrm{HDM}$, can be tested.

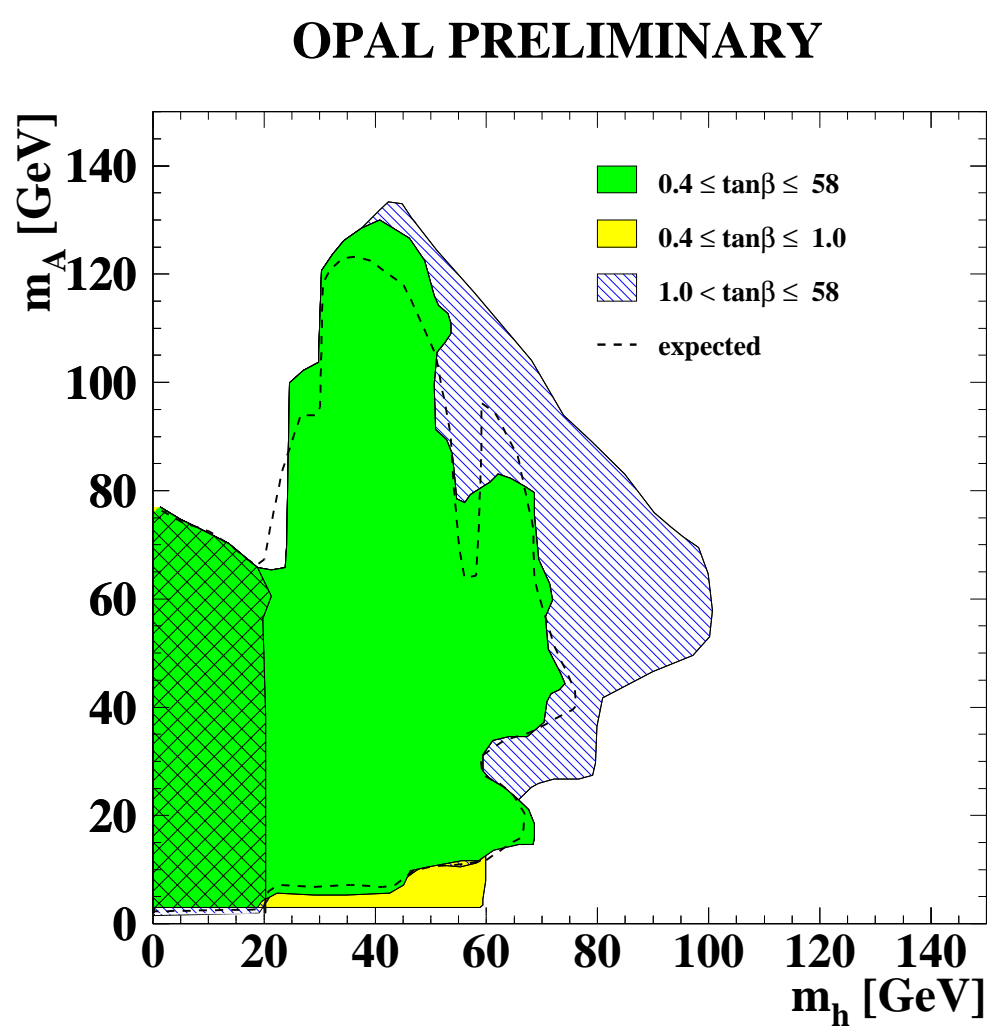

Figure 4: Regions excluded at $95 \% \mathrm{CL}$ in the $\left(m_{h}, m_{A}\right)$ plane, for any $\alpha$, and $\beta$, in the ranges $0.4 \leq \tan \beta \leq$ 58.0 (dark grey area), $0.4 \leq \tan \beta \leq 1.0$ (light grey area) and $1.0 \leq \tan \beta \leq 58.0$ (hatched area). Constraints from direct Higgs boson searches at $\sqrt{s}=M_{Z}$ [21], and from the total $Z$ boson width determination [22] are used in the combination. The exclusion from applying only these two constraints is shown in the cross-hatched region. The expected exclusion corresponding to the dark grey area is shown as a dashed line.

\section{Constraints on MSSM large $\mu$ scenarios [2]}

Higgs bosons can also have suppressed couplings to $b$-quarks in the MSSM once higher order loop corrections are included. This was shown to occur for large values of $\tan \beta$ and of the Higgs mixing parameter, $\mu$. A specific example was designed as an illustration in the LEP-2 context, and was proposed as one of three benchmark scenarios to be tested. For any choice of $m_{A}$ or $\tan \beta$ with the parameters specified ${ }^{6}$, one of the two neutral CP-even Higgs bosons always had a mass $\leq 108 \mathrm{GeV} / \mathrm{c}^{2}$ within kinematic reach at LEP-2, and

\footnotetext{
${ }^{6}$ In the specified "large $\mu$ " benchmark scenario, model parameters were scanned setting $M_{S U S Y}=400 \mathrm{GeV} / \mathrm{c}^{2}, \mu=1$ $\mathrm{TeV} / \mathrm{c}^{2}, M_{2}=400 \mathrm{GeV} / \mathrm{c}^{2}, m_{\tilde{g}}=200 \mathrm{GeV} / \mathrm{c}^{2}, 4 \leq m_{A} \leq 400 \mathrm{GeV} / \mathrm{c}^{2}$ and $X_{t}=-300 \mathrm{GeV} / \mathrm{c}^{2}$.
} 
a sizeable production cross-section, but could have a strongly suppressed coupling to $b$-quarks. Thanks to the bounds provided by the flavour independent $h Z$ search described in section 2, this particular scenario is excluded ${ }^{7}[6]$.

\section{Conclusions and prospects}

Searches for neutral Higgs bosons performed without exploiting $b$-tagging have enabled to reduce the modeldependence of the results in important ways, by not restricting their applicability to models with standard SM or MSSM-like Higgs boson couplings to $b$-quark pairs.

In the search for $h Z$ production, the combined flavour independent bounds, although preliminary and not fully optimised, were almost as strong as in the corresponding SM search. This was a surprise given the strong emphasis given to $b$-tagging in Higgs boson searches throughout the LEP-2 period, but can be understood in retrospect, from the lower background present once the $Z$ boson mass range (populated by the very signal-like $Z Z$ component) is overcome, something which was not anticipated to be easy at the start of LEP-2.

In the search for $h A$ production, although all the data have yet to be exploited and combined, results obtained so far by individual collaborations allowed to improve significantly the exclusion in the kinematically accessible mass range, and, by combining with results from other Higgs boson searches, to provide very general constraints on Type II 2HDMs.

Several applications of these searches to specific scenarios with suppressed $B R(h \rightarrow b \bar{b})$ were also possible. Composite models of the kind described in [3], although not covered in detail here, can for instance be excluded by the flavour independent bounds on $h Z$ production obtained, over the full mass range in which Higgs boson decays into gluon pairs are dominating in this model (i.e. up to $m_{h} \simeq 110 \mathrm{GeV} / \mathrm{c}^{2}$ ). The particular "large- $\mu$ " MSSM scenario, proposed to be tested at LEP-2 as a benchmark to illustrate cases giving rise to Higgs bosons with suppressed couplings to $b \bar{b}$ (together with masses and production rates well within reach at LEP-2) could also be excluded.

In year 2002, the final performance and full combination of this set of searches will be pushed, to maximize the sensitivity for all tested Higgs masses kinematically accessible at LEP-2, in as model independent a way as possible.

\section{Acknowledgements}

This presentation was given on behalf of the ALEPH, DELPHI, L3 and OPAL collaborations, and the LEP Higgs Working Group (LHWG). I would like to thank members of the flavour independent Higgs search teams in the four collaborations for the material and help provided, and for the efficient work done recently within the LHWG sub-group responsible for flavour independent combinations. I would also like to thank Pamela Ferrari, Marcel Stanitzki and Ivo van Vulpen for useful comments on this write-up.

\section{References}

[1] J. F. Gunion et al., The Higgs Hunter's Guide, Addison-Wesley Publishing Company, 1990

[2] M. Carena et al., Suggestions for improved benchmark scenarios for Higgs-boson searches at LEP2, hep-ph/9912223.

\footnotetext{
${ }^{7}$ It should be noted, however, that this result can probably not be considered as a general exclusion of MSSM scenarios with suppressed Higgs boson couplings to $b \bar{b}$ in the mass range accessible to LEP-2, as sets of model parameters different from those chosen here could lead to larger than $108 \mathrm{GeV} / \mathrm{c}^{2}$ and/or smaller production rates. A more general parameter scan would be required to identify potentially remaining unexcluded zones.
} 
M. Carena et al., Phys. Rev. D 60 (1999) 075010

W. Loinaz and J. D. Wells, Phys. Lett. B 445 (1998) 178

M. Carena et al., Nucl. Phys. B586 (2000) 92

[3] X. Calmet and H. Fritzsch, Phys. Lett. B 496 (2000) 190

[4] P. Colas, Twelve Years of Higgs Hunting at LEP, These Proceedings.

[5] The LEP Working Group for Higgs Boson searches, Search for the Standard Model Higgs bosons at $L E P$, Contribution to the EPS conference in Budapest, Hungary, 2001, ALEPH 2001-066, DELPHI 2001-113, L3 Note 2699, OPAL PN-479, LHWG note 2001-03.

[6] The LEP Working Group for Higgs Boson searches, Search for the neutral Higgs boson of the MSSM: Preliminary combined results using LEP data collected up to $209 \mathrm{GeV}$, Contribution to the EPS conference in Budapest, Hungary, 2001, ALEPH 2001-057, DELPHI 2001-114, L3 Note 2700, OPAL TN-699, LHWG note 2001-04.

[7] ALEPH Collaboration, A flavour independent search for the Higgsstrahlung process in e+e-collisions at centre-of-mass energies from 189 to $209 \mathrm{GeV}$, Contribution to the EPS conference in Budapest, Hungary, 2001, ALEPH 2001-021.

[8] DELPHI Collaboration, Generalised search for hadronic decays of Higgs bosons with the DELPHI detector at LEP-2, Contribution to the EPS conference in Budapest, Hungary, 2001, DELPHI 2001-070.

[9] L3 Collaboration, Flavour Independent Search for Hadronically Decaying Higgs Boson in Higgsstrahlung Process at $\sqrt{s}$ up to $209 \mathrm{GeV}$, Contribution to the EPS conference in Budapest, Hungary, 2001, L3 Note 2693.

[10] OPAL Collaboration, Searches for Higgs Bosons in Extensions to the Standard Model in e+e-Collisions at the Highest LEP Energies, OPAL PN-472,

OPAL Collaboration, Model Independent Searches for Scalar Bosons with the OPAL Detector at LEP, OPAL PN-449.

[11] OPAL Collaboration, Two Higgs Doublet Model Interpretation of Neutral Higgs Boson Searches up to the Highest LEP Energies, OPAL PN-475.

[12] OPAL Collaboration, Eur. Phys. J. C18 (2001) 425-445.

[13] L3 Collaboration, Search for Production of Neutral Higgs Scalars in $e^{+} e^{-}$Annilihations at LEP, Contribution to the ICHEP conference in Osaka, Japan, 2000, L3 Note 2576.

[14] The LEP Working Group for Higgs Boson searches, Flavour Independent Search for Hadronically Decaying Neutral Higgs Bosons at LEP, Contribution to the EPS conference in Budapest, Hungary, 2001, ALEPH 2001-058, DELPHI 2001-118, L3 Note 2703, OPAL TN-700, LHWG note 2001-07.

[15] D. Zer-Zion, Beyond the Standard Model Higgs Searches at LEP (part A), Proceedings of the EPS conference in Budapest, Hungary, 2001, PRHEP-hep2001/144.

[16] A. L. Read, in CERN Report 2000-005, p. 81 (2000).

[17] A. Raspereza, Private Communication.

[18] T. Junk, Nucl. Inst. Meth. A434 (1999) 435-443. 
[19] V.F. Obraztsov, Nucl. Instr. Meth. A316 (1992) 388.

[20] P. Janot, in CERN Report 96-01, Vol. 2, p. 309.

[21] OPAL Collaboration, Phys. Lett. B268 (1991) 122.

[22] The LEP Collaborations ALEPH, DELPHI, L3 and OPAL, the LEP Electroweak Working Group and SLD Heavy Flavour and Electroweak Groups, for Higgs Boson searches, CERN-EP-021 (2001). 